\title{
Health Care System in India: A Review on How Prepared we are for Covid-19
}

\author{
Swapnil Jain \\ Medical Intern, Jawaharlal Nehru Medical College,Datta Meghe Institute of \\ Medical Sciences, Wardha, Sawangi (M),Maharashtra, India. \\ Corresponding author email: swapnil.ritisha@gmail.com
}

\section{ABSTRACT}

COVID-19 emerged as the deadliest pandemic around the world. As of September 2020 second week the world registered 28 million cases with deaths nearing to one million mark. Talking about country specific India stands at second position after the United States of America with almost 4.4 million cases, adding around 80 thousand cases daily. The exponential rise of cases seriously tested the capacity of healthcare system. In India, the healthcare system was overwhelmed and underprepared. In India large population is out of social sector schemes and insurance coverage which keeps the sword of going into poverty any time hanging on their head. The more talked about data or figure of mortality rate or case fatality rate (CFR), in India, which is around $1.7 \%$ as of September 2020. Converting it into numbers after seeing huge population number can give some chills. Further the age-wise data nuances suggest that it is quite underestimated. The model test, trace and treat which is followed by most of the countries is also extensively followed in India. But the test part is also a cause of concern. Considering the changing demographics of India, a thorough planning seemed necessary to cater the future needs. This paper tried to review and outline the Indian scenario of COVID-19.

KEY WORDS: HEALTHCARE SYSTEM, INDIA, COVID-19, HEALTH INFRASTRUCTURE, VACCINE, PREPAREDNESS, PANDEMIC.

\section{INTRODUCTION}

Initially an outbreak turned epidemic. Later World Health Organization declared it a pandemic in second week of March 2020, assessing its severity of spread (Dushyant Bawiskar, 2020). As of September 2020 second week the world registered 28 million cases with deaths nearing to one million mark. Talking about country specific India stands at second position after the United States of America with almost 4.4 million cases, adding around 80 thousand cases daily. Despite one of the harshest and strictest lockdown ever imposed, the surge in cases after partial relaxation is a cause of concern (Gopalan HS,

Biosc Biotech Res Comm P-ISSN: 0974-6455 E-ISSN: 2321-4007

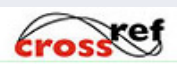

Identifiers and Pagination

Year: 2021 Vol: 14 No (6) Special Issue

Pages: 242-246

This is an open access article under Creative

Commons License Attribn 4.0 Intl (CC-BY).
DOI: $h t t p: / / d x$.doi.org/10.21786/bbrc/14.6.50
Misra A. 2020). The unforeseen situation created by the pandemic and lack of health infrastructure magnifies the already ailing healthcare sector.

In India health is a state subject meaning primary responsibility lies with the states. Yet union intervenes and formulates the policies which can be taken over by states. India spends a mere of 3.89\% of its Gross Domestic Product (GDP). Which is far less as compared to suggested value of around 6\%. The dream of universal health coverage (UHC) is also in wait to fulfill but present condition needs severe overhaul to accommodate the demands of UHC. In addition, the low penetration of secondary and tertiary care centers to the rural areas which accounts maximum population is creating the urban-rural divide. Slowly the pandemic is shifting its base to tier- 2 and tier- 3 cities and all the way to the villages which will be overwhelmed with only few cases (Kumar A, 2020). The non-pharmacological measures undertook by the authorities such as lockdown, physical distancing etc. has been successful for buying some

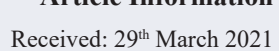

ccepted after revision: $27^{\text {th }}$ May 2021 
time to boost up the health infrastructures around the country.

The humongous population, a challenge in itself in these condition, cannot be confined to their homes as the question of livelihood arose (Chetterje P. 2020). The gradual opening of the lockdown has seen exponential rise sometimes 90000 cases per day has a silver lining of low mortality rate. Also it can be related to less virulence of the virus and more early reporting of the cases. By far the geographical reach of the virus is somewhat contained in urban areas but as gradual easing of the restrictions and festive season in near future would be alarming bell for the rural healthcare systems (Chatterjee $\mathrm{K}$, et al 2020).

Health Infrastructure: India's healthcare is system is composed of public as well as private sector. In which public sector mainly caters to rural medical services demand and private sectors which constitutes from clinics to hospital chains mainly serves the urban and resourceful people. Data on the Health Management Information System (HMIS) portal of the Ministry of Health and Family Welfare shows that the Number of Primary Health Centers (PHCs), Community Health Centers (CHCs), Sub-District/Divisional Hospitals(SDHs), District Hospitals(DHs) and beds, India, State/UT wise as uploaded by the State - Total 7,39,024 beds in 37,725 facilities. Number of Govt. Hospitals and beds in Rural and Urban Areas, India, State/UT wise, as available in the Publication National Health Profile 2018- 279588 beds in rural and 431173 beds in urban facilities (Balsari S et al 2020).

The availability of beds should not be confused with other critical infrastructure like oxygen support system and ventilators etc. which are crucial in COVID-19 cases. Although from available data only 4-5 percent patients of COVID-19 requires ventilator facility it is still a big number. But the Intensive Care Unit requiring patients are higher in number, which (ICU) are not available or fully functional at the health center. Also uniform regulatory standard across the spectrum is absent which hinders in quality health services. The conversion of stadiums, open grounds, railway coaches into isolation wards has only exposed the urban healthcare system too where congested communities and slum dwellings put huge pressure on infrastructure especially in this type of pandemic. Decentralization of the urban located secondary and tertiary health care units to rural clusters is the need of the hour. As access to healthcare is also a problem as restriction of movement was imposed but are gradually easing up. At least every primary health center should be able to attend the longstanding chronic illness cases with sufficient ICU beds and oxygen support system.

Comparing the hardest hit countries such as Italy and Spain, the health care infrastructure in these two European economies where health care penetration is high and almost everybody is covered under health scheme and insurance run by governments (which is not the case in India), even their health infrastructure were severely overwhelmed and overburdened.

On the contrary, In India large population is out of social sector schemes and insurance coverage which keeps the sword of going into poverty any time hanging on their head. The more talked about data or figure of mortality rate or case fatality rate (CFR), in India, which is around $1.7 \%$ as of September 2020. Converting it into numbers after seeing huge population number can give some chills. Further the age-wise data nuances suggest that it is quite underestimated. The model test, trace and treat which is followed by most of the countries is also extensively followed in India. But the test part is also a cause of concern. India is testing around 1.2 million samples each day which is screened in 1623 labs across the country. But dissection of testing into most trusted RT-PCR tests and Rapid antibodies test is interesting to see as later has to be once again confirmed by the former one.

Lack of availability of beds, beds with ventilators and oxygen support, knowledge of all these to the patient is seriously hampering our efforts. A proper database which can be single point availability of all beds types and distance can be very beneficial in saving the golden time of treatment. As more early you treat less spread will occur. This lack of beds anecdotes spreading mouth to mouth is resulting in unwanted consequence of patients with mild or no symptoms not turning to the health center. And relying on the so called home remedies which can be fatal in two ways. First for the patient itself as it can worsen the condition if patient have co-morbidities. Second the patient cannot be monitored and hence is free to roam resulting in more human to human contact which can accentuate the spread.

A Case for Universal Health Coverage: Right to health is a fundamental right under article 21 of the constitution. Therefore everyone has a right to get a minimum of good quality health services whether he or she is poor or rich, resides in urban or rural area. It is a part and parcel of right to life. Universal Health Coverage (UHC) will ensure the health services to all the citizens regardless of income level, gender, social status, caste or religion. And knowing the huge population size of the India, it is quite herculean task but is extremely necessary. UHC will guarantee preventive, curative, rehabilitative care to all. Government can be provider in such case but the private sector participation is also necessary for larger penetration. Although there are some challenges of severe underfunding of public health infrastructure, rising costs of health services in private sector, inadequate quality and absence of widespread coverage, there is always a way out.

In this difficult time of pandemic there is already a reduction in health coverage as the focus is currently on pandemic. Once this mess is cleared then brainstorming on universal health coverage is must so that most of the cases can be detected at initial stages and treated in primary centers rather prolonging it to tertiary care 
centers which is costly affair. Integrating pharmacy sector which in India is the largest manufacturer of most affordable vaccines for preventable diseases with the core health sector can also immensely benefit the overall outcome.

Divergence of all the resources to fight COVID-19: The pandemic has forced the health sector to singularly focus on COVID-19 containment. This has led to allocation of most of the resources for containing pandemic spread. The sector which is already in dire need of investment and partially fragile has lot bear on itself. Another aspect of this is that the people suffering from other chronic illnesses such as diabetes, hypertension, renal failure etc. have been completely neglected. The postponement of various surgeries points out these trends. There is a need of proper data on urgent and non-urgent surgeries and modification according to the data.

Otherwise the toll from neglecting the priorities from non-COVID19 patients will exceed the COVID-19 toll. For example, the patients of dialysis and diabetes needs regular intervention otherwise the condition worsens and the patient can be needed the ICU bed which are already less in number due to diversion. The data by Indian Council of Medical Research (ICMR) shows that there are only 70000 thousand Intensive Care Units (ICU)beds available in which number of beds having ventilator support is even less. Of which most of the beds are reserved for the COVID-19 cases which in turns left out the patients having non-COVID-19 disease such as heart ailments and others which also requires the same bed type.

Another less talked impact is on universal immunization program for children. Around 90-95 \% of immunization occurs through government undertaken Universal Immunization Program. Polio is one of the praiseworthy campaigned runs by India. The goal of completely eradicating tuberculosis by 2025 needs reassessment. It also severely hampers the ongoing efforts to contain another communicable disease. Authorities claims that India may see rise in TB cases in India. During current scenario much of the public health machinery is diverted to wards the COVID-19 pandemic. Further such as NPI's such as lockdown, common fear of contracting disease averted the parents from going the healthcare center. The effects of these events will only be seen after the pandemic is over but government should start the doorstep delivery of the universal vaccines and medicine which is also a difficult task considering low human resource in health sector. During the World Immunization Week (24 to 30April 2020) the WHO had warned about resurgence of preventable diseases which have vaccines available (Somashekhar, S. P., et al 2020).

Human Resource: Healthcare professionals are the frontline warriors who are fighting the disease for the masses. Utmost care and support with proper equipment's are must to ensure they can work in stress free environment. But in the initial phase of the pandemic, the lack of proper personal protective equipment's (PPE's) were felt and this exposes some professionals to the virus. This lowers the number of doctors available as India's doctor to population ratio is far less than WHO'S prescribes ratio of one doctor for 1000 peoples. India has one doctor for around 1445 people. Studies and surveys suggest that in India there is only one government doctor for every 10189 people which according to WHO should be one government doctor for one thousand people. Which points towards deficiency of 600000 doctors. Furthermore, the nurse to patient ratio is 1:483 implying two million nurse shortages.

Overall, India needs two times the current strength of the doctors and three times the nurses and four times the paramedical staffs. Also, the allopathic doctors who treats the patient was also less. This overwhelms the current human resource with more burden as they have to treat more patient than before. Doctors and other health care professionals are working long and strenuous hours wearing PPE's which makes it difficult for them to even dispense the waste material from their body. Further the constant fear of contracting the disease to their family members is always present at the back of their mind. This creates unease as they cannot meet their loved ones due to this fear. Despite these difficulties the health workers which are our frontline warriors are fighting the spread of the virus and doing their job.

The issue of lack manpower can be addressed by employing a short duration bridge course. The professionals from allied health care sector can be trained such a that a basic level and preliminary examining can be done by them so that the burden on core health care professionals reduces and issue of man power also can be addressed. Also authority's needs to check on quackery, bogus doctors especially in rural areas where awareness is low. Also, another cause of concern is infection rate among health workers. It is seen that positivity rate which is the rate of number person tested positive out of number persons tested is double in health workers than the average of countries positivity rate. Long work hours due to shortage of staffs is also taxing on the health workers be it doctors, nurses, ward boys or paramedics.

Vaccine Trials: As India mulls for a multidisciplinary approach through its new education policy announced recently the research and development department should also be part of healthcare infrastructure. The integration between the doctors and researchers will only results in better outcomes. The research and development are also lacking in India as there is low public as well as private investments. As far as vaccine on COVID-19 is concern the Serum Institute of India has partnered with university Of Oxford on the Vaccine Named 'Covishield'. It has entered the phase- 2 trials which will be followed by phase- 3 trials. In addition, the recent development of Sputnik-V by Russian researchers also has been monitored and Russian authorities shared their trials data with Indian authorities. The country of the size of India, it really needs to think about the vaccine as soon as possible as only it will guarantee the further deterioration of the current situation be it number of cases or number 
of deaths due to COVID-19. But we need to accept that still it is a long road to reliable and safe vaccine till then the present healthcare system hasevolved daily according to changing circumstances.

As of latest bite Serum Institute which partnered with University of oxford which is developing the leading candidate in COVID-19 vaccine race has halted the phase trials in India due to some adverse observations seen in United Kingdom's trial, which further implies to strengthen the health infrastructure. On the other hand, study published in lancet shows the string immune system response generated by Sputnik-V vaccine from Russia. Although the trials were conducted on extremely small group, further studies and research needed for testing efficacy of vaccine. The saying of Haste makes waste goes perfect with vaccine development as even minor adverse impact can affect large number of people forget about the contraindications.

Demographic Change: As the demography of 1.35 billion people changes, there is a strong need to think on the old age care infrastructure for future. We should be ready for another unprecedented health crisis. Contingencies are best faced when contingency measures are taken. We know that one can never accurately predict the crisis but building a resilient and robust infrastructure which can survive at least a crisis of this scale would suffice. Further one of the main reasons of India having one of the lowest mortality rates is young population. In India there are more young population than older ones mean more persons having strong natural immune system response. But as the time passes and fertility rate stabilizes the average age would be increasing and more old persons will depend upon less young population. This will overwhelm the health sector so better be ready and there is no good time to start now.

Rising average age is also a cause of concern as it will also increase the cases of non-communicable disease (NCD'S) which are largely attributed to life style changes. COVID-19 pandemic gives us a chance to relook the strategies that are packed and redraw the new one to deal such situation. According to 2011 census India have 104 million elderly peoples which will become 173 million in 2026 reported by United Nations (UN). Merely increasing lifespan without having good quality of life with lesser ailments will alone would not suffice. (Somashekahar et al 2020) reviewed the ASI's Consensus Guidelinesto be followed in Covid 19. (Spoorty et al 2020) have reviewed about mental health problems faced by healthcare workers due to the Covid-19 pandemic. Risk factors of respiratory health issues in this region are evident from the previous studies (Taksande, et al 2016, Dhar, Raja,et al 2019 and Salampuria, et al 2019). Early detection can cure preventable diseases which accounts most of the deaths and taken casually as age related illness and avoided.

More coverage of doctors and allied professionals will be needed for this to bear the huge ailing population. Geriatric care should be strengthened more than today.
The challenge for the health sector is to promote healthy life style, improve access to and utilization of health care. The future diseases of would cause mainly due to lifestyle changes. Which is also a case for current scenario. Rising awareness and technological advancements will lead to more people demanding better health facilities. The factor of cost of healthcare is also a challenge before the system.

Although there are lot of problems present in the healthcare system, we need to find our way out with the help of lessons taught by the COVID-19. The pandemic showed the mirror to the healthcare systems of the world including India. A country advancing to one and half billion population must think and design flexible and resilient policies and infrastructure not just for now but for the future needs of the changing demographics (Nibudey, A., and S. Vidya Baliga. 2020, Parveen, S., and S. Jain 2020 and Parwe, S.D., et al 2020). A mix of private and public sector participation through involving non-governmental organization can immensely benefit and help in achieving the goal of good quality health care for all.

Further strengthening the health professionals with necessary equipment's and guidance to deal catastrophe of such scale is necessary. Right training of health professionals to deal with any eventualities. Proper dissemination of information right from the top will ensure people taking right decisions and not fall into the pray of Infodemic (Parwe, S.D., et al 2020, Pasari, A.S. et al 2020, Pate, M.Y., et al 2020 and Quazi, A.A., and M. Patil. 2020). Studies by (Prasad et.al. 2020, Sharma et.al. 2020, Singh et.al.2020 and Bawiskar et.al. 2020) addressed the emerging needs and measures in respective sections of health specialties. Vaccine in near future is still only in developmental stage, parallel investment in current health sector public as wells as private will ensure the manageable conditions in future. Preventive care is also a major factor which can lessen the burden of the healthcare system.

\section{CONCLUSION}

To ensure every citizen gets a minimum of necessary health services hassle free adequate augmentation of the services is must to do. There is also a need to improve access to health services for vulnerable sections, women which goes beyond the normal services. Only the healthy population will serve as demographic dividend which should be supported by well-equipped infrastructure.

\section{REFERENCES}

Balsari, S., Sange, M. and Udwadia, Z., 2020. COVID-19 care in India: the course to self-reliance. The Lancet Global Health, 8(11), pp.e1359-e1360.

Bawiskar, N., Andhale, A., Hulkoti, V., Acharya, S. and Shukla, S., 2020. Haematological Manifestations of Covid-19 and Emerging Immunohaematological Therapeutic Strategies. Journal of Evolution of Medical and Dental Sciences, 9(46), pp.3489-3495. 
Bawiskar, D., Phansopkar, P. and Gotmare, A.V., 2020. Covid-19 facets: Pandemics, curse and humanity. International Journal of Research in Pharmaceutical Sciences, 11(Special Issue 1).

Buheji, M. and Buhaid, N., 2020. Nursing human factor during COVID-19 pandemic. International Journal of Nursing, 10(1), pp.12-24.

Chatterjee, K., Chatterjee, K., Kumar, A. and Shankar, S., 2020. Healthcare impact of COVID-19 epidemic in India: A stochastic mathematical model. Medical Journal Armed Forces India, 76(2), pp.147-155.

Chetterje, P., 2020. Gaps in India's preparedness for COVID-19 control. The Lancet Infectious Diseases, 20(5), p.544.

Dhar, R., Mohan, M., D’souza, G., Rajagopalan, S., Singh, V., Jindal, A., Ghewade, B., Joshi, G., Sahasrabuddhe, T., Gami, S. and Nair, G., 2017. Phenotype characterization of non cystic fibrosis bronchiectasis in India: baseline data from an Indian bronchiectasis registry. In B 103. CLINICAL STUDIES IN BRONCHIECTASIS, IMMUNODEFICIENCY, AND DRUG INDUCED LUNG DISEASE (pp. A4726-A4726). American Thoracic Society.

Dhar, R., Singh, S., Talwar, D., Mohan, M., Tripathi, S.K., Swarnakar, R., Trivedi, S., Rajagopala, S., D’Souza, G., Padmanabhan, A. and Baburao, A., 2019. Bronchiectasis in India: results from the European multicentre bronchiectasis audit and research collaboration (EMBARC) and respiratory research network of India registry. The Lancet Global Health, 7(9), pp.e1269e1279.

Gopalan, H.S. and Misra, A., 2020. COVID-19 pandemic and challenges for socio-economic issues, healthcare and national programs in India. Diabetes \& Metabolic Syndrome: Clinical Research \&t Reviews.

Islam, M.S., Sarkar, T., Khan, S.H., Kamal, A.H.M., Hasan, S.M., Kabir, A., Yeasmin, D., Islam, M.A., Chowdhury, K.I.A., Anwar, K.S. and Chughtai, A.A., 2020. COVID19-related infodemic and its impact on public health: A global social media analysis. The American Journal of Tropical Medicine and Hygiene, 103(4), p.1621.

Izzetti, R., Nisi, M., Gabriele, M. and Graziani, F., 2020. COVID-19 transmission in dental practice: brief review of preventive measures in Italy. Journal of dental research, 99(9), pp.1030-1038.

Kumar, A., Nayar, K.R. and Koya, S.F., 2020. COVID-19: Challenges and its consequences for rural health care in India. Public Health in Practice, 1, p.100009.

Latchoumi, T.P., Ezhilarasi, T.P. and Balamurugan, K., 2019. Bio-inspired weighed quantum particle swarm optimization and smooth support vector machine ensembles for identification of abnormalities in medical data. SN Applied Sciences, 1(10), pp.1-10.

Parveen, S. and Jain, S., 2020. Pathophysiologic Enigma of COVID-19 Pandemic with Clinical Correlates. International Journal of Current Research and Review,
12, pp.33-37.

Parwe, S.D., Ingle, A.S., Nisargandha, M.A. and Rathi, B., 2020. Healthcare workers novel coronavirus (NCOVID 19) life-threatening situation during the pandemic. International Journal of Research in Pharmaceutical Sciences, 11(Special Issue 1).

Parwe, S.D., Nisargandha, M.A. and Thakre, R., 2020. Role of convalescent plasma therapy in new Coronavirus disease (nCOVID-19): A review. International Journal of Research in Pharmaceutical Sciences, 11(Special Issue 1).

Pasari, A.S., Bhawane, A., Balwani, M.R., Tolani, P., Ramteke, V. and Deshpande, N., 2020. Knowledge about COVID-19 and Practices among Hemodialysis Technicians in the COVID-19 Pandemic Era. International journal of nephrology, 2020.

Pate, B.S., Yeola, M.E., Gawande, A., Singh, A.K. and Tayade, H.A., 2020. Best Practices for Endoscopic Procedures in Covid-19 Pandemic. Journal of Evolution of Medical and Dental Sciences, 9(49), pp.3760-3767. Prasad, N., Bhatt, M., Agarwal, S.K., Kohli, H.S., Gopalakrishnan, N., Fernando, E., Sahay, M., Rajapurkar, M., Chowdhary, A.R., Rathi, M. and Jeloka, T., 2020. The adverse effect of COVID pandemic on the care of patients with kidney diseases in India. Kidney international reports, 5(9), pp.1545-1550.

Salampuria, S., Jajoo, S. and Acharya, S., 2019. Acute respiratory distress syndrome during pregnancy and post-partum-a case series with spectrum of near miss to mortality. Journal of Evolution of Medical and Dental Sciences-JEMDS, 8, pp.3724-26.

Singh, K.T., Mishra, G., Shukla, A.K., Behera, S., Tiwari, A.K., Panigrahi, S. and Chhabra, K.G., 2020. Preparedness among dental professionals towards COVID-19 in India. The Pan African Medical Journal, 36.

Singh, N., Anjankar, A.P. and Garima, S., 2020. The Urgent Need to Understand Covid-19 Associated Coagulopathies and the Significance of Thrombotic Prophylaxis in Critically Ill Patients. Journal of Evolution of Medical and Dental Sciences, 9(33), pp.2381-2386.

Somashekhar, S.P., Shivaram, H.V., Abhaham, S.J., Dalvi, A., Kumar, A., Gode, D., Misra, S., Jain, S.K., Prasad, C.R.K. and Pillarisetti, R.R., 2020. ASI's consensus guidelines: $\mathrm{ABCs}$ of what to do and what not during the COVID-19 pandemic

Spoorthy, M.S., Pratapa, S.K. and Mahant, S., 2020. Mental health problems faced by healthcare workers due to the COVID-19 pandemic-A review. Asian journal of psychiatry, 51, p.102119

Taksande, A.M. and Yeole, M., 2016. Risk factors of Acute Respiratory Infection (ARI) in under-fives in a rural hospital of Central India. Journal of Pediatric and Neonatal Individualized Medicine (JPNIM), 5(1), pp.e050105-e050105 